GUEST EDITORIAL

\title{
Understanding the genetic basis of human health and disease: Role of molecular genetics in diagnosis and prognostication
}

Human genetics is the study of inheritance as it occurs in human beings. It encompasses several overlapping areas, including cytogenetics, molecular genetics, biochemical genetics, genomics, clinical genetics, developmental genetics, population genetics and genetic counselling. Genes can be the common factor underlying the qualities of most human-inherited traits. The study of human genetics can be useful, as it can answer questions about human nature, understand diseases and development of effective disease treatment, and contribute to understanding genetics of human life. DNA contains instructions for everything our cells do, from conception until death. Studying the human genome allows us to explore fundamental details about ourselves. ${ }^{[1]}$

Genomics refers to the field of genetics focused on structural and functional studies of the genome. A genome is all the DNA contained within an organism or a cell, including nuclear and mitochondrial DNA. The human genome is the total collection of all genes in a human being contained in the human chromosomes and composed of $>3$ billion nucleotides. In April 2003, in arguably the biggest scientific advance of the last 2 decades, the Human Genome Project (HGP) was able to sequence all the DNA in the human genome, and to discover that the human genome was composed of $\sim 20000$ protein-coding genes.

Human reproduction shows inheritance patterns, both of the type discovered by Mendel (autosomal inheritance) and of sexlinked inheritance. As we are dealing with people rather than experimental organisms that can be studied in the laboratory, human geneticists must resort to scrutinising records in the hope that information about inheritance has shown itself in the pedigree analysis of the phenotype of parents and their children. As is often the case, nature provides the best laboratory. A member of a family who first comes to the attention of a geneticist is called the proband. Usually, the phenotype of the proband is exceptional in some way (e.g. characteristic facies or short stature). The geneticist is then able to trace the history of the phenotype in the proband back through the history of the family and draws a family tree or pedigree. In the study of genetic disorders, four general patterns of inheritance are distinguishable by pedigree analysis: autosomal recessive, autosomal dominant, $\mathrm{X}$-linked recessive, and $\mathrm{X}$-linked dominant.

A glossary of terms is set out in Table 1 .

\section{The Human Genome Project}

The HGP, the international quest to understand the genomes of humans and other organisms, was planned to provide information on a wide range of basic questions: how many genes we have, how cells work, how living organisms evolved, how single cells develop into complex creatures, and what exactly happens when we become unwell. Importantly, it was hoped that the HGP would not merely answer questions regarding our molecular composition, but also usher in a new era of molecular medicine, with precise novel approaches to prevent, diagnose and treat human disease. ${ }^{[2]}$

The HGP began in the USA in 1990, when the National Institutes of Health and the US Department of Energy joined forces with international partners to decipher the massive amount of information contained in our genomes. The HGP began with a set of ambitious goals, but has exceeded nearly all its targets. HGP scientists have produced an increasingly detailed series of maps that help geneticists navigate through human DNA and have mapped and sequenced the genomes of important experimental organisms. The HGP began transforming biology as soon as it started, because

\section{Table 1. A glossary of terms}

\begin{tabular}{|c|c|}
\hline Chromosomes & $\begin{array}{l}\text { Thread-like structure of nucleic acids and proteins found in the nucleus of most living cells, carrying genetic information } \\
\text { in the form of genes }\end{array}$ \\
\hline DNA & $\begin{array}{l}\text { Deoxyribonucleic acid is a long molecule made of two twisting, paired strands. Each strand is made of four chemical units, } \\
\text { called nucleotide bases, strung together in a precise order, just as letters string together to make specific words. The bases are } \\
\text { adenine (A), guanine (G), cytosine (C) and thymine (T). The bases on opposite strands pair specifically; an A always pairs } \\
\text { with a T, and a C always with a G. Each such pair is called a base pair of DNA }\end{array}$ \\
\hline Gene & Functional unit of DNA. Each of the human chromosomes contains hundreds to thousands of genes \\
\hline Genome & All the DNA contained within an organism or a cell, including nuclear and mitochondrial DNA \\
\hline Genomics & The field of genetics concerned with structural and functional studies of the genome \\
\hline Human genetics & The study of inheritance as it occurs in human beings \\
\hline Mutation & Changes in DNA spelling that can prevent proteins from functioning normally and cause health problems \\
\hline Protein & $\begin{array}{l}\text { Proteins comprise essential parts of tissues and guide chemical reactions in living things. They are made of } 20 \text { different } \\
\text { building blocks called amino acids. The DNA sequence of a gene determines the amino acid sequence of the protein that } \\
\text { gene encodes. The amino acid sequence of the protein is, in turn, responsible for the protein's shape and function }\end{array}$ \\
\hline RNA & $\begin{array}{l}\text { Ribonucleic acid is chemically similar to DNA, except that it is single stranded, not double stranded; it contains the base } \\
\text { uracil (U) instead of thymine (T); it can migrate out of the nucleus. The sequences of most RNA molecules are translated } \\
\text { to make proteins }\end{array}$ \\
\hline SNP & $\begin{array}{l}\text { Single-nucleotide polymorphisms or one-letter variations in the DNA sequence. SNPs contribute to differences among } \\
\text { individuals. The majority have no effect, others cause subtle differences in countless characteristics, such as appearance, } \\
\text { while some affect the risk for certain diseases }\end{array}$ \\
\hline
\end{tabular}


the information it generated was disseminated rapidly through unrestricted, public databases and has fuelled the unmatched pace of discovery into the genetic basis of many human conditions.

\section{The genetic basis of health and disease}

Genes are made up of DNA, which encodes all genetic information. Almost all human cells contain 23 pairs of chromosomes; each chromosome contains a molecule of DNA with hundreds to thousands of genes arrayed along it. Genes usually code for proteins, the diverse molecules that perform a wide variety of specialised tasks in the cells. ${ }^{[2]}$ Alterations in our genes are responsible for $>5000$ clearly hereditary diseases, such as hypertrophic cardiomyopathy, sickle cell disease, cystic fibrosis and Huntington disease, to name a few. The altered sequence of many other genes influences the development of common illnesses that arise through the interaction of genes with the environment.

Linking a gene with a specific disease was historically a slow, imprecise and painstaking process before the advent of the HGP and molecular genomics. In 1989, only 4 genes were definitively linked with disease through analysis of heredity. Ten years later, the list included $>100$ genes. Consider two gene hunts, 8 years apart: in 1989, scientists found the gene for cystic fibrosis after a 9-year search; 8 years later, a gene for Parkinson disease was mapped in only 9 days, and precisely described within 9 months.

Almost all the 100 trillion cells in the human body contain a copy of the entire human genome, the complete set of genetic instructions necessary to build a human being, finely tuned by various epigenetic mechanisms to control our biology. Knowing the DNA sequence of a gene reveals the basic structure of the protein that gene encodes. Scientists can sometimes deduce the 3-dimensional shape and function of the protein as well. Often, they can classify the protein because of similarities to other proteins.

\section{The role of human genetics in medicine}

Examining how a gene is mutated in an individual has multiple utility: ${ }^{[2]}$

Diagnosis. Genetic analysis can now classify conditions such as colon cancer, skin cancer and lung cancer into finer categories, which is important because improved genotypic classification allows more precise and efficacious therapies. Detailed molecular classification is also emerging in cardiovascular disease, mental health and other areas where genetic underpinning of human health is becoming better understood.

Pharmacogenomics. The practice of tailoring drugs for patients, whose individual response can be predicted by genetic fingerprinting, is fast gaining traction. For example, in cancer patients facing chemotherapy, not only can this field allow for prediction of an individual's metabolic responses to drugs that can minimise side-effects and maximise efficacy, but genetic fingerprinting of their tumour can inform which chemotherapy choices are most likely to be effective. Better understanding of genetics promises a future of precise, customised medical treatments, i.e. precision medicine.

Prognosis. More accurate diagnosis allows more precise prediction about the natural history and course of a disease. For instance, the genetic work-up in a patient with familial hypercholesterolaemia or prostate cancer can inform us of the likely risk of adverse outcomes in those individuals. Molecular genetic screening also allows patients and doctors to weigh the risks and benefits of different treatments.

Prevention. Once scientists figure out which DNA sequence changes in a gene can cause disease, healthy people can be tested to see whether they risk developing specific conditions and appropriate preventive strategies can be employed early. For example, in many countries babies are routinely screened for phenylketonuria, a metabolic disorder that prevents the breakdown of phenylalanine, which is toxic to the nervous system. In the past, children with the condition became severely intellectually disabled but the screening programme identifies children with the enzyme deficiency, allowing them to grow normally on a diet that strictly avoids phenylalanine. Molecular approaches to both newborn screening and adult risk stratification are rapidly being refined and applied.

Carrier screening. There are some genetic conditions where people who have mutated genes will not be ill themselves, but they can pass the disease to their offspring. For instance, carrier testing for sickle cell disease, which is a major cause of morbidity and mortality in Africa, has been available and widely used for years.

Gene-based therapy. Replacing a misspelled gene with a functional gene has long been an appealing idea but has remained challenging to implement. Small groups of patients have undergone gene-replacement therapy in clinical trials for more than 2 decades, but this remains largely an experimental treatment. Recently, gene-based therapies have been shown to be efficacious in many conditions such as adenine deaminase deficiency, Duchenne muscular dystrophy, sickle cell anaemia and HIV. For years, effort has been put into designing drugs based on an understanding of how genes work and what exactly happens at the molecular level to cause disease. For example, it is possible to administer a small molecule that interacts with the protein - as many drugs do - and change its behaviour. Protein kinase inhibitors, used for the management of chronic myelogenous leukaemia, were one of the first examples of such gene-based therapy, using a rationally designed drug target based on the understanding of the molecular pathways resulting from a fusion protein caused by a translocation of chromosomes 9 and 22 .

In this issue of CME, Krause ${ }^{[3]}$ provides an update on novel genetic testing platforms, and reviews their advantages and limitations. Fieggen et al. ${ }^{[4]}$ discuss a pragmatic approach to the genetic diagnosis of developmental delay and tools that are appropriate and cost-effective in South African settings. Finally, Scott et al. ${ }^{[5]}$ suggest a genetic approach to the understanding and diagnosis of suspected hereditary breast and colorectal cancer.

It is our hope that the readers will find this issue to be interesting and instructive.

\section{Karen J Fieggen}

Division of Human Genetics, Department of Medicine, Faculty of Health Sciences, Groote Schuur Hospital and University of Cape Town, South Africa

\section{Ntobeko A B Ntusi}

Division of Cardiology, Department of Medicine, Faculty of Health Sciences, University of Cape Town and Groote Schuur Hospital; Cape Universities Body Imaging Centre, Faculty of Health Sciences, University of Cape Town; and Hatter Institute for Cardiovascular Research in Africa, Department of Medicine, Faculty of Health Sciences, University of Cape Town, South Africa ntobeko.ntusi@uct.ac.za
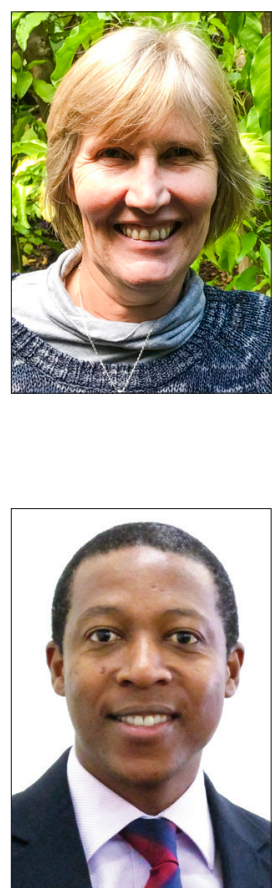
1. Kork BR. Introduction to human genetics. In: Robertson D, Williams GH, eds. Clinical and Translational Science. Principles of Human Research. 2nd ed. London, UK: Elsevier, 2017:281-311. 2. Genetics: The future of medicine. The Human Genome Project - exploring our molecular selves. https://www.genome.gov/pages/educationkit/images/nhgri/pdf (accessed 8 March 2019).

Krause A. New genetic technologies: Advantages and limitations. S Afr Med J 2019;109(4):207-209. https://doi.org/10.7196/SAMJ.2019.v109i4.13990

4. Fieggen KJ, Lambie LA, Donald KA. Investigating developmental delay in South Africa: A pragmatic approach. S Afr Med J 2019;109(4):210-213. https://doi.org/10.7196/SAMJ.2019.v109i4.13991
5. Scott CJ, Schoeman M, Urban MF. Cancer genetics: An approach to suspected hereditary breast and colorectal cancer. S Afr Med J 2019;109(4):214-218. https://doi.org/10.7196/SAMJ.2019. v109i4.13992

S Afr Med J 2019;109(4):204-206. DOI:10.7196/SAMJ.2019.v109i4.14037 